\title{
SOME NEW SEPARATION AXIOMS IN GENTOP
}

\author{
KHEDIDJA DOURARI AND SAMI LAZAAR
}

\begin{abstract}
In this paper, we introduce some new separation axioms in the category of generalized topological spaces. Some characterizations of these axioms are given. Morphisms rendered invertible by the $T_{0}$-reflection in GenTop are entirely determined. Finally, some known results in the category Top are deduced.
\end{abstract}

\section{INTRODUCTION}

Topologists have introduced the notion of generalized topological spaces as a generalization of topological spaces to study a large variety of properties for sets. Today this class of spaces is used to solve many problems in different areas such as complex modelling, image analysis, graph theory and economical modelling (for more information see [4] and [6]).

The construction of the $T_{0}$-reflection in the category TOP is given by Herrlich and Strecker in [9]. After that, some authors have been interested in the $T_{0}$-reflection in other categories as a generalization of the first one. At this time, we can cite Richmond and Künzi in the category PREORDTOP whose objects are preorder-topological spaces $(X, \tau, \leq)$ and continuous increasing maps as arrows [10].

In $[2,5,6]$ Mashhor, Allam, Mahmoud, Khedr and Császár studied the concept of generalized topologies. Let $X$ be a nonempty set. A generalized topology (briefly GT) on $X$ is a collection $\mu$ of subsets of $X$ containing the empty set stable under union. If $\mu$ is a $\mathrm{GT}$ on $X$, the pair $(X, \mu)$ is called a generalized topological space (briefly GTS). A map $f:\left(X, \mu_{X}\right) \longrightarrow\left(Y, \mu_{Y}\right)$ between GTS's is said to be continuous if $f^{-1}(G) \in \mu_{X}$ for all $G \in \mu_{Y}$. The category with GTS's as objects and continuous maps as arrows is denoted by GenTop. Equivalently, GenTop can be defined as the category with all pairs $(X, \gamma)$ as objects where $X$ is a nonempty set and $\gamma$ is a map from the power set $\mathcal{P}(X)$ into itself such that:

(i) For all subsets $A, B$ of $X$, we have $A \subseteq B$ implies $\gamma A \subseteq \gamma B$. ( $\gamma$ is called monotonic)

(ii) For all subsets $A$ of $X$, we have $A \subseteq \gamma A$. ( $\gamma$ is enlarging)

(iii) For all subsets $A$ of $X$, we have $\gamma A=\gamma \gamma A$. ( $\gamma$ is idempotent)

That is, GenTop is the category whose objects are the family $(X, \gamma)$ of all clspaces (i.e, $X$ equipped with a monotonic and enlarging operator) with idempotent

$M S C$ (2010): primary 54A05, 54D10, 18A40; secondary 39.

Keywords: Generalized topological spaces, $T_{0}$-reflection.

The research was supported by the laboratory LATAO, code: LR11ES12. 
closure operator and arrows the family of cl-continuous maps. Recall that a map $q:(X, \gamma) \longrightarrow(Y, \delta)$ between two cl-spaces is said to be cl-continuous if $\gamma q^{-1}(B) \subseteq$ $q^{-1}(\delta B)$ for all subsets $B$ of $Y$, or equivalently $q(\gamma A) \subseteq \delta q(A)$ for all subsets $A$ of $X$. If in addition $\gamma(\emptyset)=\emptyset,(X, \gamma)$ will be called a strong GTS. A strong GTS satisfying $\gamma(A \cup B)=\gamma(A) \cup \gamma(B)$ is a topological space and, in this case, $\gamma$ is called a Küratowsky operator.

In [3], the authors defined some new separation axioms named $T_{(i, j)}$ as follows.

Definition 1.1. Let $i, j$ be two integers such that $0 \leq i<j \leq 2$. A topological space $X$ is said to be a $T_{(i, j)}$-space if its $T_{i}$-reflection is a $T_{j}$-space. Thus, there are three new types of separation axioms; namely, $T_{(0,1)}, T_{(0,2)}$ and $T_{(1,2)}$.

Since the property $T_{D}$ is not reflective in Top (recall that a $T_{D}$-space is a topological space in which every point is locally closed), the previous definition can be generalized by the below.

Definition 1.2. Let $F$ be a (covariant) functor from Top to itself, and $P$ be a topological property. An object $X$ of Top is said to be a $T_{(F, P)}$-object if $F(X)$ satisfies the property $P$.

In this paper we study the link between these new separation axioms and the category of GTS's. Consequently, some new separation axioms are introduced and studied. So, many results of [3] are clarified and deduced. In the first section, we recall some classic separation axioms and their characterizations used in the next sections. The second section is devoted to introducing and characterizing the new separation axioms $T_{(0,1)}, T_{(0, D)}$ and $T_{(0,2)}$ in the category GenTop. In the third section maps rendered invertible by the reflector $T_{0}$ in GenTop are given.

In this paper all considered GTS's are assumed to be strong.

\section{Some Classic Separation Axioms in GenTop}

In this section, we study the properties of some classic separation axioms in GenTop.

We begin by recalling some notions of generalized topological spaces. Let $(X, \gamma)$ be a GTS. The members of $\operatorname{Im} \gamma=\{\gamma(A): A \subseteq X\}=\{A \subseteq X: \gamma(A)=A\}$ are called $G$-closed sets and their complements are called $G$-open sets. We denoted by $i_{\gamma}$ the generalized interior in $(X, \gamma)$ defined by $i_{\gamma}(A)=X \backslash \gamma(X \backslash A)$ for all subsets $A$ of $X$. It is easy to verify that a subset $A$ of $X$ is $G$-open if and only if $i_{\gamma}(A)=A$. A subset $A$ of $(X, \gamma)$ is said to be $G$-locally closed if it is an intersection of a $G$-closed and a $G$-open subset of $X$, or equivalently if there exists a $G$-open subset $i_{\gamma}(B)$ of $X$ such that $A=\gamma(A) \cap i_{\gamma}(B)$.

Proposition 2.1. Let $(X, \gamma)$ be a GTS. A subset $A$ of $X$ is $G$-locally closed if and only if $\gamma(A) \backslash A$ is $G$ closed.

Definition 2.2. Let $(X, \gamma)$ be a GTS. Then, $(X, \gamma)$ is said to be a $T_{0}$ GTS if, for each two distinct points $x, y \in X$, there exists a G-closed subset of $X$ containing one of these points, but not the other.

The following result is immediate. 
Proposition 2.3. Let $(X, \gamma)$ be a GTS. Then, the following statements are equivalent:

(i) $(X, \gamma)$ is a $T_{0}$ GTS;

(ii) for every $x, y \in X$, we have $\gamma(\{x\})=\gamma(\{y\})$ implies $x=y$.

Definition 2.4. Let $(X, \gamma)$ be a GTS. Then, $(X, \gamma)$ is said to be a $T_{1}$ GTS if, for each $x \in X, \gamma(\{x\})=\{x\}$.

Proposition 2.5. Let $(X, \gamma)$ be a GTS. Then, the following statements are equivalent:

(i) $(X, \gamma)$ is a $T_{1} G T S$;

(ii) for each pair of distinct points $x, y$ of $X$, there exists a $G$-closed subset of $X$ containing $x$ but not $y$;

(ii) for each $x \in X,\{x\}=\bigcap\{U \mid U \in G(x)\}$, where $G(x)$ is the collection of all $G$-open subsets of $X$ containing $x$.

Definition 2.6. Let $(X, \gamma)$ be a GTS. Then, $(X, \gamma)$ is said to be a $T_{D}$ GTS if for each $x \in X,\{x\}$ is $G$-locally closed.

The following result characterizes $T_{D}$ GTS's.

Proposition 2.7. Let $(X, \gamma)$ be a GTS. Then, the following statements are equivalent:

(i) $(X, \gamma)$ is a $T_{D} G T S$

(ii) for each $x \in X, \gamma(\{x\}) \backslash\{x\}$ is G-closed.

Definition 2.8. Let $(X, \gamma)$ be a GTS. Then, $(X, \gamma)$ is said to be a $T_{2}$ GTS if, for each two distinct points $x, y \in X$, there exist two disjoint G-open subsets of $X$ containing respectively $x$ and $y$.

Proposition 2.9. Let $(X, \gamma)$ be a GTS. Then, the following statements are equivalent :

(i) $(X, \gamma)$ is a $T_{2} G T S$;

(ii) for each $x \in X,\{x\}=\bigcap\{\gamma(U) \mid U \in G(x)\}$.

Remark 2.10. The following implications are immediate in GenTop:

$T_{2} \Longrightarrow T_{1} \Longrightarrow T_{D} \Longrightarrow T_{0}$.

Every topological space is a generalized topological space and it is well known that the converses of the previous implications do not hold for topological spaces. Thus, the converses do not hold in GenTop. But in the following, we give some non-trivial examples (non-topological spaces) illustrating these situations.

Examples 2.11. (1) A $T_{0}$ GTS need not be a $T_{D}$ GTS. Indeed, let $X=$ $\{a, b, c\}$ and $\gamma$ be a map from the power set $P(X)$ into itself defined by $\gamma(\{a\})=\{a, b\}, \gamma(\{c\})=\{a, c\}$ and $\gamma(A)=X(A \neq \emptyset)$ otherwise. Then, $(X, \gamma)$ is a $T_{0}$ GTS. However, $\gamma(\{a\}) \backslash\{a\}=\{b\}$ is not $G$-closed. So $(X, \gamma)$ is not a $T_{D}$ GTS.

(2) A $T_{D}$ GTS need not be a $T_{1}$ GTS. Indeed, let $X=\{a, b, c\}$ and $\gamma$ be a map from the power set $P(X)$ into itself defined by $\gamma(\emptyset)=\emptyset, \gamma(\{a\})=$ $\gamma(\{a, c\})=\{a, c\}, \gamma(\{b\})=\{b\}, \gamma(\{c\})=\{c\}, \gamma(\{a, b\})=\gamma(X)=X$, 
$\gamma(\{b, c\})=\{b, c\}$. Then, $(X, \gamma)$ is a $T_{D}$ GTS. However, $\gamma(\{a\}) \neq\{a\}$. So $(X, \gamma)$ is not a $T_{1}$ GTS.

(3) A $T_{1}$ GTS need not be a $T_{2}$ GTS. Indeed, let $X=\{a, b, c\}$ and $\gamma$ be a map from the power set $P(X)$ into itself defined by $\gamma(\emptyset)=\emptyset, \gamma(\{x\})=\{x\}$ for all $x \in X, \gamma(A)=X$, for all other subsets $A$ of $X$. Then, $(X, \gamma)$ is a $T_{1}$ GTS. However, $\{a\} \neq \bigcap\{\gamma(U) \mid U \in G(a)\}$. So $(X, \gamma)$ is not a $T_{2}$ GTS.

\section{Some new Separation axioms in GenTop}

We begin by recalling the $T_{0}$-reflection of a GTS. Let $(X, \gamma)$ be a GTS and define the binary relation $\sim$ on $X$ by $x \sim y$ if and only if $\gamma(\{x\})=\gamma(\{y\})$. Then, $\sim$ is an equivalence relation on $X$. Let $\hat{X}$ be the quotient set $X \backslash \sim$ and $h_{X}$ the canonical map from $X$ onto $\hat{X}$. We can define the operator $\hat{\gamma}$ from $\mathcal{P}(\hat{X})$ to itself by $\hat{\gamma}(B)=h_{X}\left(\gamma\left(h_{X}^{-1}(B)\right)\right)$, for every $B \subseteq \hat{X}$. Then, $(\hat{X}, \hat{\gamma})$ is a $T_{0}$ GTS.

The full subcategory GenTop of $_{0}$ all $T_{0}$ GTS's is reflective in GenTop. Hence, the inclusion functor I : GenTop $\mathbf{G}_{0} \hookrightarrow$ GenTop has a left adjoint

$$
T_{0}: \text { GenTop } \longrightarrow \text { GenTop }_{0}
$$

which assigns to each $G T S(X, \gamma)$ the $T_{0}$ induced GTS $(\hat{X}, \hat{\gamma})$ and to each morphism $q:(X, \gamma) \longrightarrow(Y, \delta)$ in GenTop a unique morphism $\hat{q}:(\hat{X}, \hat{\gamma}) \longrightarrow(\hat{Y}, \hat{\delta})$ defined by $\hat{q}\left(h_{X}(x)\right)=h_{Y}(q(x))$.

Such a situation can be illustrated by the following diagram:

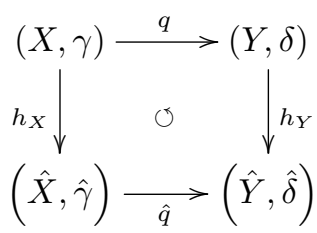

Now, let us recall that a continuous map $q:(X, \gamma) \longrightarrow(Y, \delta)$ between GTS's is said to be a quasihomeomorphism if the induced map $\varphi_{q}: \operatorname{Im} \delta \longrightarrow \operatorname{Im} \gamma$ defined by $\varphi_{q}(\delta(B))=q^{-1}(\delta(B))$ is bijective. Equivalently, $\varphi_{q}^{\prime}: A \longrightarrow q^{-1}(A)$ induces a bijective map from the family of $G$-open subsets of $Y$ to the family of $G$-open subsets of $X$.

As in Top it is clear that if $f, g$ and $h$ are three continuous maps in GenTop such that $f \circ g=h$ whenever any two of them are quasihomeomorphisms, so is the third one.

Now, since $h_{X}$ in GenTop is constructed in the same way as in Top (just replacing $\gamma(\{x\})$ by $\overline{\{x\}})$, one can deduce easily the following remark:

Remark 3.1. Let $(X, \gamma)$ be a GTS. Then, the induced map $h_{X}:(X, \gamma) \longrightarrow$ $(\hat{X}, \hat{\gamma})$ is an onto quasihomeomorphism.

The following proposition gives some properties of onto quasihomeomorphisms.

Proposition 3.2. Let $q:(X, \gamma) \longrightarrow(Y, \delta)$ be a continuous onto map between GTS's. Then, the following statements are equivalent:

(1) $q$ is a quasihomeomorphism; 
(2) $q$ is closed and, for each subset $A$ of $X$, we have $q^{-1}(q(\gamma(A)))=\gamma(A)$;

(3) $q$ is open and, for each subset $A$ of $X$, we have $q^{-1}\left(q\left(i_{\gamma}(A)\right)\right)=i_{\gamma}(A)$.

Proof. (1) $\Rightarrow(2)$ Let $A=\gamma(A)$ be a $G$-closed subset of $X$. Since $q$ is a quasihomeomorphism, there exists a subset $B$ of $Y$ such that $A=\gamma(A)=q^{-1}(\delta(B))$. Now, since $q$ is onto, $q(\gamma(A))=q\left(q^{-1}(\delta(B))\right)=\delta(B)$. Therefore, $q$ is a closed map and $q^{-1}(q(\gamma(A)))=q^{-1}(\delta(B))=\gamma(A)$.

$(2) \Rightarrow(1)$ Let $A$ be a subset of $X$. Then, by hypothesis, $q(\gamma(A))=\delta(q(\gamma(A)))$ and thus $q^{-1}(\delta(q(\gamma(A))))=\gamma(A)$. Therefore, $\varphi_{q}$ is onto. Now let $B$ and $B^{\prime}$ be two subsets in $Y$ such that $\varphi_{q}(\gamma(B))=\varphi_{q}\left(\gamma\left(B^{\prime}\right)\right)$. Then, $\gamma(B)=q\left(q^{-1}(\gamma(B))\right)=$ $q\left(q^{-1}\left(\gamma\left(B^{\prime}\right)\right)\right)=\gamma\left(B^{\prime}\right)$. Thus, $\varphi_{q}$ is one-to-one, hence, $q$ is a quasihomeomorphism.

In the same way, we can deduce $(1) \Longleftrightarrow(3)$.

Definition 3.3. Let $(X, \gamma)$ be a GTS. Then, $(X, \gamma)$ is said to be a $T_{(0,1)}$ GTS if its $T_{0}$-reflection is a $T_{1}$ GTS.

The following result gives a characterisation of $T_{(0,1)}$ GTS's.

Theorem 3.4. Let $(X, \gamma)$ be a GTS. Then, the following statements are equivalent:

(1) $(X, \gamma)$ is a $T_{(0,1)} G T S$;

(2) For each $x \in X, \gamma(\{x\})=h_{X}^{-1}\left(h_{X}(\{x\})\right)$;

(3) For each $x, y \in X, x \in \gamma(\{y\}) \Longrightarrow y \in \gamma(\{x\})$;

(4) For each $x, y \in X$ such that $\gamma(\{x\}) \neq \gamma(\{y\})$, there exists a G-closed subset of $X$ containing $x$ but not $y$;

(5) For each $x \in X$ and each subset $A$ of $X$ such that $\gamma(\{x\}) \cap \gamma A \neq \emptyset, x \in \gamma A$;

(6) For each subset $A$ of $X$ and each $x \in i_{\gamma}(A), \gamma(\{x\}) \subseteq i_{\gamma}(A)$;

(7) For each $x \in X, \gamma(\{x\})=\bigcap\{U \mid U \in G(x)\}$.

Proof. $(1) \Rightarrow(2)$ Let $x \in X$. Since $(\hat{X}, \hat{\gamma})$ is $T_{1}$, we have:

$$
h_{X}\left(\gamma\left(h_{X}^{-1}\left(\left\{h_{X}(x)\right\}\right)\right)\right)=\left\{h_{X}(x)\right\} \text {. }
$$

Now, we can see easily that:

$$
\gamma(\{x\})=\gamma(\{y \in X: \gamma(\{y\})=\gamma(\{x\})\}) .
$$

Hence:

$$
\gamma(\{x\})=\gamma\left(h_{X}^{-1}\left(\left\{h_{X}(x)\right\}\right)\right)=h_{X}^{-1}\left(h_{X}(\gamma(\{x\}))\right) .
$$

Then, $h_{X}(\gamma(\{x\}))=h_{X}(x)$ and thus $\gamma(\{x\})=h_{X}^{-1}\left(\left\{h_{X}(x)\right\}\right)$.

$(2) \Rightarrow(3) x \in \gamma(\{y\})=h_{X}^{-1}\left(h_{X}(\{y\})\right) \Longrightarrow h_{X}(x) \in\left\{h_{X}(y)\right\} \Longrightarrow h_{X}(x)=$ $h_{X}(y) \Longrightarrow y \in h_{X}^{-1}\left(h_{X}(\{x\})\right)=\gamma(\{x\})$.

$(3) \Rightarrow(4)$ Let $x, y$ be two points such that $\gamma(\{x\}) \neq \gamma(\{y\})$. By hypothesis, $y \notin \gamma\{x\}$ and thus $\gamma(\{x\})$ is a $G$-closed subset containing $x$ but not $y$.

(4) $\Rightarrow(5)$ Let $x \in X$ and $A \subseteq X$ such that $\gamma(\{x\}) \cap \gamma(A) \neq \emptyset$. Fix a point $y$ in $\gamma(\{x\}) \cap \gamma(A)$. Then, $y \in \gamma(\{x\})$. Now, since $y \in \gamma(A), \gamma(\{y\}) \subseteq \gamma(A)$.

If $x$ is not in $\gamma(A)$, then $\gamma(\{x\}) \neq \gamma(\{y\})$ and thus by hypothesis there exists $B=\gamma(B)$ containing $x$ but not containing $y$. So, $[y \in \gamma(\{x\}) \subseteq \gamma(B)$ and $y \notin$ $\gamma(B)]$ which is impossible.

$(5) \Rightarrow(6)$ Let $i_{\gamma}(A)$ be a $G$-open subset of $(X, \gamma)$ and $x \in i(A)$. Then, $x \notin$ $\gamma(X \backslash A)$. Therefore, $\gamma(\{x\}) \cap \gamma(X \backslash A)=\emptyset$, so $\gamma(\{x\}) \subseteq i_{\gamma}(A)$. 
$(6) \Rightarrow(7)$ By hypothesis the first inclusion is immediate.

Conversely, note that $t \in \gamma(\{x\})$ if and only if any $G$-open subset containing $t$ also contains $x$.

Hence, let $t \in \bigcap\{U \mid U \in G(x)\}$. So every $G$-open subset containing $x$ also contains $t$ and thus $x \in \gamma(\{t\}) \subseteq \bigcap\{U \mid U \in G(t)\}$. This means that any $G$-open set containing $t$ contains $x$. Therefore, $t \in \gamma(\{x\})$.

$(7) \Rightarrow(1)$ Let $x$ and $y$ be two points in $X$ such that $h_{X}(x) \neq h_{X}(y)$. Then, $\gamma(\{x\}) \neq \gamma(\{y\})$.

Let us check that $x \notin \gamma(\{y\})$. So if not $x \in \bigcap\{U \mid U \in G(y)\}$ and, consequently, $x$ belongs to every $G$-open subset containing $y$ then $y \in \gamma(\{x\})$, immediately implying that $\gamma(\{x\})=\gamma(\{y\})$, which is impossible.

Then, $x \notin \gamma(\{y\})=\bigcap\{U \mid U \in G(y)\}$. So there exists a $G$-open subset $U$ of $X$, containing $y$ but not $x$. Finally, $h_{X}(U)$ is a $G$-open subset of $\hat{X}$ containing $h_{X}(y)$ but not $h_{X}(x)$.

Remark 3.5. The [3, Theorem 3.5] is a particular case of Theorem 3.4 if we consider a topological space $X$ and define $\gamma$ by $\gamma(A)=\bar{A}$, for any subset $A$ of $X$.

Definition 3.6. Let $(X, \gamma)$ be a GTS. Then, $(X, \gamma)$ is said to be a $T_{(0, D)}$ GTS if the $T_{0}$-reflection $(\hat{X}, \hat{\gamma})$ is a $T_{D}$ GTS.

The following result gives a characterization of $T_{(0, D)}$ GTS's.

Theorem 3.7. Let $(X, \gamma)$ be a GTS. Then, the following statements are equivalent:

(i) $(X, \gamma)$ is a $T_{(0, D)} G T S$;

(ii) for each $x \in X, \tau(\{x\})=\gamma(\{x\}) \backslash\{y \in X \mid \gamma(\{x\})=\gamma(\{y\})\}$ is a G-closed subset of $(X, \gamma)$.

Proof. $(X, \gamma)$ is $T_{(0, D)} \Longleftrightarrow(\hat{X}, \hat{\gamma})$ is $T_{D}$ GTS

$$
\begin{array}{ll}
\Longleftrightarrow \quad \forall x \in X, \hat{\gamma}\left(\left\{h_{X}(x)\right\}\right) \backslash\left\{h_{X}(x)\right\} \text { is G-closed subset of }(\hat{X}, \hat{\gamma}) \\
\Longleftrightarrow \quad \forall x \in X, h_{X}^{-1}\left(\hat{\gamma}\left(\left\{h_{X}(x)\right\}\right)\right) \backslash h_{X}^{-1}\left(\left\{h_{X}(x)\right\}\right) \\
& \text { is G-closed subset of }(X, \gamma) \\
\Longleftrightarrow \quad & \forall x \in X, \gamma\left(h_{X}^{-1}\left(\left\{h_{X}(x)\right\}\right)\right) \backslash\{y \in X: \gamma(\{y\})=\gamma(\{x\})\} \\
& \text { is G-closed subset of }(X, \gamma) \\
\Longleftrightarrow \quad & \forall x \in X, \gamma(\{x\}) \backslash\{y \in X: \gamma(\{y\})=\gamma(\{x\})\} \\
& \text { is G-closed subset of }(X, \gamma) \\
& \forall x \in X, \tau(\{x\}) \text { is G-closed in }(X, \gamma) .
\end{array}
$$

Remark 3.8. The [3, Theorem 3.3] is a particular case of Theorem 3.7 if we consider a topological space $X$ and define $\gamma$ by $\gamma(A)=\bar{A}$, for any subset $A$ of $X$.

Definition 3.9. Let $(X, \gamma)$ be a GTS. Then, $(X, \gamma)$ is said to be a $T_{(0,2)}$ GTS if the $T_{0}$-reflection $(\hat{X}, \hat{\gamma})$ is $T_{2}$. 
Lemma 3.10. Let $q:(X, \gamma) \longrightarrow(Y, \delta)$ be an onto quasihomeomorphism. Then, for each $x \in X, \bigcap\left\{\gamma\left(q^{-1}(V)\right) \mid V \in G(q(x))\right\}=q^{-1}(\bigcap\{\delta(V) \mid V \in G(q(x))\})=$ $\bigcap\{\gamma(U) \mid U \in G(x)\}$.

Theorem 3.11. Let $(X, \gamma)$ be a GTS. Then, the following statements are equivalent:

(1) $(X, \gamma)$ is a $T_{(0,2)}$ GTS ;

(2) For each $x, y \in X$ such that $\gamma(\{x\}) \neq \gamma(\{y\})$, there exist two disjoint $G$-open subsets $i_{\gamma}(A)$ and $i_{\gamma}(B)$ of $X$ containing $x$ and $y$, respectively;

(3) For each $x \in X, \gamma(\{x\})=\bigcap\{\gamma(U) \mid U \in G(x)\}$.

Proof. (1) $\Rightarrow(2)$ Let $x, y \in X$ be such that $\gamma(\{x\}) \neq \gamma(\{y\})$. Then $h_{X}(x) \neq$ $h_{X}(y)$. So, by hypothesis, there exist two disjoint $G$-open subsets $i_{\hat{\gamma}}(C)$ and $i_{\hat{\gamma}}(D)$ with $h_{X}(x) \in i_{\hat{\gamma}}(C)$ and $h_{X}(y) \in i_{\hat{\gamma}}(D)$. Therefore, $h_{X}^{-1}\left(i_{\hat{\gamma}}(C)\right)$ and $h_{X}^{-1}\left(i_{\hat{\gamma}}(D)\right)$ are two disjoint $G$-open subsets of $X$ containing $x$ and $y$, respectively.

$(2) \Rightarrow(1)$ Let $x, y \in X$ be such that $h_{X}(x) \neq h_{X}(y)$. Then, $\gamma(\{x\}) \neq \gamma(\{y\})$. Hence, there exist two disjoint $G$-open subsets $i_{\gamma}(A)$ and $i_{\gamma}(B)$ of $(X, \gamma)$ such that $x \in i_{\gamma}(A)$ and $y \in i_{\gamma}(B)$. Since $h_{X}$ is a quasihomeomorphism, there are two $G$-open subsets $i_{\hat{\gamma}}(C)$ and $i_{\hat{\gamma}}(D)$ of $(\hat{X}, \hat{\gamma})$ such that $h_{X}^{-1}\left(i_{\hat{\gamma}}(C)\right)=i_{\gamma}(A)$ and $h_{X}^{-1}\left(i_{\hat{\gamma}}(D)\right)=i_{\gamma}(B)$ and thus $i_{\hat{\gamma}}(C)$ and $i_{\hat{\gamma}}(D)$ are two disjoint $G$-open subsets containing $h_{X}(x)$ and $h_{X}(y)$, respectively.

$(1) \Rightarrow(3)$ Let $x \in X$. Since $(X, \gamma)$ is $T_{(0,2)}$, we have $\left\{h_{X}(x)\right\}=\bigcap\{\hat{\gamma}(V) \mid V \in$ $\left.G\left(h_{X}(x)\right)\right\}$. Now, since every $T_{(0,2)}$ GTS is $T_{(0,1)}$,

$$
\begin{aligned}
\gamma(\{x\}) & =h_{X}^{-1}\left(\left\{h_{X}(x)\right\}\right) \\
& =\bigcap\left\{h_{X}^{-1}(\hat{\gamma}(V)) \mid V \in G\left(h_{X}(x)\right)\right\} \\
& =\bigcap\left\{\gamma\left(h_{X}^{-1}(V)\right) \mid V \in G\left(h_{X}(x)\right)\right\} \\
& =\bigcap\{\gamma(V) \mid V \in G(x)\} .
\end{aligned}
$$

$(3) \Rightarrow(1)$ First, we show that $(X, \gamma)$ is a $T_{(0,1)}$ GTS. Let $x \in X$. We are aiming to prove that $\gamma(\{x\})=\bigcap\{U \mid U \in G(x)\}$. It is easily seen that $\bigcap\{U \mid U \in$ $G(x)\} \subseteq \gamma(\{x\})$. Conversely, let $y \in \gamma(\{x\})$. Then, for each $G$-open subset $i_{\gamma}(A)$ of $(X, \gamma)$ containing $y, x \in i_{\gamma}(A)$. So, by hypothesis, $x \in \gamma(\{y\})$. Therefore, $\gamma(\{x\}) \subseteq \bigcap\{U \mid U \in G(x)\}$. Thus, $\gamma(\{x\})=\bigcap\{U \mid U \in G(x)\}$; that is $(X, \gamma)$ is $T_{(0,1)}$. This implies that $\gamma(\{x\})=h_{X}^{-1}\left(\left\{h_{X}(x)\right\}\right)$. Applying Lemma 3.10, we have $\left\{h_{X}(x)\right\}=\bigcap\left\{\hat{\gamma}(V) \mid V \in G\left(h_{X}(x)\right)\right\}$, so that $(X, \gamma)$ is a $T_{(0,2)} \operatorname{GTS}$.

Remark 3.12. The [3, Theorem 3.12] is a particular case of Theorem 3.11 if we consider a topological space $X$ and define $\gamma$ by $\gamma(A)=\bar{A}$, for any subset $A$ of $X$.

\section{Morphisms Rendered invertible by the $T_{0}$-Reflection in GenTop}

For any functor $F: \mathbf{C} \longrightarrow \mathbf{D}$ between two categories, sometimes, the family of all arrows in $\mathbf{C}$ rendered invertible by $F$ has important applications $[12,13]$. In this section we are interested in morphisms of GenTop rendered invertible by the functor $\mathbf{T}_{\mathbf{0}}$. To this end, we need to introduce some definitions. 
Definition 4.1. Let $q:(X, \gamma) \longrightarrow(Y, \delta)$ be a continuous map between GTS's.

(1) $q$ is said to be topologically onto if, for each $y \in Y$, there exists an $x \in X$ such that $\delta(\{y\})=\delta(\{q(x)\})$.

(2) $q$ is said to be topologically one-to-one if, for each $x, y \in X$ such that $q(x)=q(y), \gamma(\{x\})=\gamma(\{y\})$.

(3) $q$ is said to be topologically bijective if it is topologically onto and topologically one-to-one.

Example 4.2. (1) Every onto continuous map between GTS's is topologically onto.

(2) Every one-to-one continuous map between GTS's is topologically one-to-one.

In general, a topologically one-to-one map between GTS's need not be oneto-one. Let $(X, \gamma)$ be a GTS which is not $T_{0}$. The canonical onto map $h_{X}$ is topologically one-to-one but not one-to-one.

Remark 4.3. Let $q:(X, \gamma) \longrightarrow(Y, \delta)$ be a continuous map between GTS's.

(1) If $(X, \gamma)$ is $T_{0}$, then $q$ is one-to-one if and only if it is topologically one-toone.

(2) If $(Y, \delta)$ is $T_{0}$, then $q$ is onto if and only if it is topologically onto.

The following result gives a characterisation of morphisms in GenTop rendered invertible by the reflector $T_{0}$.

Theorem 4.4. Let $q:(X, \gamma) \longrightarrow(Y, \delta)$ be a continuous map between GTS's. Then, the following statements are equivalent:

(i) $q$ is a topologically onto quasihomeomorphism;

(ii) $\hat{q}$ is a homeomorphism.

Proof. (i) $\Rightarrow$ (ii) Since $\hat{q} h_{X}=h_{Y} q, h_{Y} \circ q$ and $h_{X}$ are two quasihomeomorphisms, $\hat{q}$ is a quasihomeomorphism.

Using Remark $4.3(2), \hat{q}$ is onto. Now let $x, y \in X$ be such that $\hat{q}\left(h_{X}(x)\right)=$ $\hat{q}\left(h_{X}(y)\right)$. Then, $\delta(\{q(x)\})=\delta(\{q(y)\})$. We are aiming to prove that $\gamma(\{x\})=$ $\gamma(\{y\})$. It suffices to show that $\gamma(\{x\}) \subseteq \gamma(\{y\})$. Let $A$ be a subset of $X$ such that $x \in i_{\gamma}(A)$. Since $q$ is a quasihomeomorphism, there exists a subset $B$ of $Y$ such that $q^{-1}\left(i_{\delta}(B)\right)=i_{\gamma}(A)$. Then, $q(y) \in i_{\gamma}(A)$; that is $y \in i_{\gamma}(A)$. So $\gamma(\{x\}) \subseteq \gamma(\{y\})$. Now, using Proposition 3.2, a bijective quasihomeomorphism is a homeomorphism and thus $\hat{q}$ is a homeomorphism.

(ii) $\Rightarrow$ (i) Clearly, $q$ is a quasihomeomorphism. It remains to be proved that $q$ is topologically onto. To this end, let $y \in Y$. Since $\hat{q}$ is onto, there exists an $x \in X$ such that $\hat{q}\left(h_{X}(x)\right)=h_{Y}(y)$, which means that $h_{Y}(q(x))=h_{Y}(y)$. Consequently, $\delta(\{q(x)\})=\delta(\{y\})$.

Acknowledgment. The authors gratefully acknowledge the many helpful corrections, comments and suggestions of the anonymous referee.

\section{REFERENCES}

[1] M. Abbassi, S. Lazaar and A. Mhemdi, T0-reflection and some separation axioms in PRETOP, Filomat, to appear. 
[2] A. A. Allam, F. H. Khedr, F.S. Mahmoud and A.S. Mashhur, On supratopological spaces, Journal of Pure and Applied Mathematics 14 (1985), 502-510.

[3] K. Belaid, O. Echi and S. Lazaar, $T_{(\alpha, \beta)}$-spaces and the Wallman compactification, International Journal of Mathematics and Mathematical Sciences 2004 (2004), 3717-3735.

[4] Á. Császár, Generalized topology, generalized continuity, Acta Mathematica Hungarica 96 (2002), 351-357.

[5] Á. Császár, Generalized open sets in generalized topologies, Acta Mathematica Hungarica 106 (2005), 53-66.

[6] Á. Császár, $\delta$ - and $\theta$-modification of generalized topologies, Acta Mathematica Hungarica 120 (2008), 275-279.

[7] A. Grothendieck, Eléments de géométrie algébrique I: Le langage des schémas, Publications Mathmatiques de l'IHÉS 4 (1960), 1-122.

[8] A. Grothendieck and J. Dieudonné, Eléments de géométrie algébrique, Die Grundlehren der mathematischen Wissenschaften 166, Springer-Verlag, New York, 1971.

[9] H. Herrlich and G. Strecker, Categorical topology - its origins as exemplified by the unfolding of the theory of topological reflections and coreflections before 1971, in: C. E. Aull and R. Lowen (eds.), Handbook of the History of General Topology, Vol. 1, Kluwer, Dordrecht, 1997, 255-341.

[10] H-P. A. Künzi and T.A. Richmond, T $T_{i}$-ordered reflections, Applied General Topology 6 (2005), 207-216.

[11] S. Lazaar and A. Mhemdi, On some properties of $T_{0}$-ordered reflection, Applied General Topology 15 (2014), 43-54.

[12] S. MacLane, Categories for the Working Mathematician, Graduate Texts in Mathematics 5, Springer, New York, 1971.

[13] M. H. Stone, Application of Boolean algebra to topology, Recueil Mathematique (Matematicheskii Sbornik) 1(43) (1936), 765-771.

Khedidja Dourari, Department of Mathematics, Faculty of Sciences of Tunis, University of Tunis El Manar, Tunisia

e-mail: mararmdourari@gmail.com

Sami Lazaar, Department of Mathematics, Faculty of Sciences of Tunis, University of Tunis El Manar, Tunisia

e-mail: salazaar72@yahoo.fr 
